\title{
MIGRACIONES INTERNACIONALES Y TEORIA SOCIAL. ALGUNAS CONSIDERACIONES
}

\section{Juan Salcedo}

El propósito de las páginas que siguen es el destacar algunos de los problemas teóricos que plantea el fenómeno de las migraciones laborales internacionales. Por migraciones laborales internacionales entenderemos el desplazamiento a otro país, por un período de tiempo de, al menos, dos años de un colectivo de trabajadores de diversos niveles de cualificación, con el propósito de realizar un trabajo remunerado. Quedan, por lo tanto, excluidas del objeto del presente artículo las migraciones políticas, "fugas de cerebros», emigraciones de jubilados, así como las migraciones estacionales o de temporada.

Aunque en los últimos años, con motivo de la crisis económica generalizada, el ritmo migratorio internacional ha disminuido de forma sensible, la cierto es que en este momento se están manifestando con gran fuerza los problemas y consecuencias de los movimientos migratorios pasados, sobre todo de los acaecidos en Europa Occidental durante el período 1960-75.

No son los movimientos de población un fenómeno reciente, ni son pocos los textos en que se han descrito, de forma más o menos afortunada, algunas 
de sus características fundamentales ${ }^{1}$. Sin embargo, es muy poco lo que se ba avanzado en el resbaladizo terreno de la interconexión entre los movimientos migratorios y la teoría de la estructura social. Así, la mayoría de los estudios al uso no pasan de ser un catálogo de pautas, comportamientos, o casos-tipo ${ }^{2}$, cuando no una búsqueda a la manera de Diógenes de un culpable que casi siempre suele ser alguno de los modernos Deus ex máchina de la sociedad actual tal como el sistema económico capitalista en su fase de capitalismo monopolista ${ }^{3}$.

Culpas o causas aparte, lo cierto es que el fenómeno migratorio existe, tanto en sociedades «avanzadas» (sean capitalistas o socialistas), como en las eufemísticamente llamadas "sociedades en vías de desarrollo" (esto es, los países subdesarrollados). Así el fenómeno urbano, tan característico de la sociedad de nuestro tiempo, ha ido parejo con un proceso de éxodo rural muy intenso cuya meta final era la gran ciudad, bien del propio país o de otro más avanzado.

De entre las múltiples posibilidades existentes, la migración de campesinos sin tierra de los países en vías de desarrollo a las ciudades superurbanizadas de los países industrializados es uno de los fenómenos humanos más destacables de nuestros días. El paso brusco y repentino de un medio cultural a otro es algo que pone a prueba la capacidad de adaptación individual y colectiva de los emigrantes que pasan, de ser individuos integrados en un sistema socioeconómico, a elementos solamente integrados en el aparato productivo de su nuevo país de residencia, aunque marginados en su estructura social; de individuos identificados ${ }^{4}$ a elementos identificables sólo por su nacionalidad o su grupo étnico. Esta pérdida de identidad sólo puede ser salvada mediante un auténtico proceso de adaptación colectiva en el sentido que los ecólogos de Chicago daban a este término.

Los procesos de adaptación colectiva de los grupos inmigrantes tienden a-reproducir la vida en las comunidades de origen; a idealizar gran parte de

t Con fechas recientes se han publicado bastantes textos en torno al problema de las migraciones en general y de las migraciones internacionales en particular. Sobre estas últimas cabe destacar el conocido libro de CASTLEs y Kosack (1973), donde los autores enfocan el problema desde una perspectiva marxista ingenua. Más radical es el estudio de la inmigración a Francia publicado por CETEDIM (1975). Una de las mejores colecciones de artículos existentes es la publicada bajo la dirección de Ph. BERNARd (1976). Como aportaciones españolas cabe destacar el libro de A. Pascual (1970) sobre el retorno de los emigrantes o los artículos de GINER y SALCEDo $(1976,1978)$ en torno a los problemas teóricos que plantea el estudio de la emigración internacional.

2 Rose, A. (1972).

${ }^{3}$ Es la postura adoptada, entre otros, por Casties y Kosack (1973) y por André Gorz (1970), quien con un magistral artículo de tres páginas ha conseguido ser uno de los autores más citados sobre el tema de las migraciones laborales en Europa Occidental.

- La palabra identificados se utiliza aquí en el sentido de propietarios de una identidad definida a los ojos de los demás y no en el de partidarios de alguna idea que es el tradicional. 
los aspectos de la vida cotidiana en esas comunidades, así como a minimizar las tensiones, conflictos y desigualdades existentes en una suerte de construcción social de un «paraíso perdido» miltoniano fuertemente idealizado y ajeno a la realidad. Frente a la nueva colectividad en que están inmersos, los inmigrantes aparecen como excesivamente rígidos y apegados a la norma, evidenciando una falta de capacidad para transgredirla; capacidad utilizada por los nativos y que forma parte de unos hábitos adquiridos a lo largo del proceso socializador seguido desde su niñez. Esta capacidad de transgresión de la norma es la que confiere a los nativos del país una válvula de escape ante los múltiples problemas con que se enfrentan en su vida cotidiana, válvula de que no disponen los colectivos inmigrantes, sometidos a lo que se podría denominar «la rigidez del desconocimiento»; precisamente esta rigidez afecta a quienes más necesitarían de un escape, como son los grupos de recién llegados ${ }^{5}$.

En las páginas que siguen voy a pasar revista a algunos de los problemas teóricos más destacados que plantea la existencia de migraciones laborales en las sociedades industriales actuales. De todos ellos, trataré de forma preferente el problema de la «integración» del inmigrante en la estructura social existente y al análisis de los cambios que los inmigrantes pueden provocar en el nuevo país de adopción; en particular me interesa entrar en la polémica existente acerca del supuesto papel mantenedor del orden social que las comunidades inmigrantes desempeñarían en la sociedad post-industrial actual. Para ello especularé con la capacidad de los nuevos inmigrados para interactuar con sus iguales en el país de adopción y sus posibilidades reales y teóricas de adaptación e integración en el mismo.

Previamente a todo ello hay que señalar algunas de las dificultades que plantea el análisis de las relaciones entre emigración y estructura social. En primer término hay que hablar de la insuficiencia del concepto de clase social al analizar la situación de la clase del trabajador inmigrante en el país de adopción. Es insuficiente porque en buena parte de los casos del inmigrante viene de sociedades con sistemas de estratificación diferentes al del capitalismo occidental, por lo que la situación de tal trabajador ha de enfocarse desde la doble perspectiva sociedad de origen-sociedad de adopción. Si bien es evidente que, desde nuestra perspectiva, es un miembro más de la clase trabajadora (o media, según los casos), no es menos cierto que en muchas ocasiones el recién llegado tiene una lógica y una percepción de su situación totalmente diferente a la nuestra; y lo cierto es que esa percepción es lo que

Este tema, de gran relevancia, no ha sido aún objeto de estudio detallado por parte de sociólogos. Existe algún informe psiquiátrico aislado (LASA y Martinez, 1970) sobre el problema de psicopatologias producidas por los procesos migratorios en el País Vasco con anterioridad a los años setenta, pero es bien poco lo que se ha avanzado en este terreno o en el más conocido del shock cultural. 
va a condicionar de forma inmediata una buena parte de sus decisiones ${ }^{6}$. En segundo término hay que proceder a una reinterpretación del concepto de integración social en un sentido menos etnocentrista al que actualmente se viene utilizando, como equivalente a asimilación cultural ${ }^{7}$. Es un hecho conocido el de que, en la mayor parte de los casos, se equipara la integración a la pérdida de la propia identidad cultural, política, lingüística, etc.

En último término, me gustaría señalar cómo el tema de las migraciones internacionales va dejando de ser un tema marginal para convertirse en problema político de primera magnitud, como veremos en los años venideros, sobre todo si la CEE tiende a consolidarse definitivamente y se produce su ampliación a doce miembros.

\section{I}

Un elemento central en el estudio del problema de la inmigración es el de la integración. El estereotipo que los estudiosos - conservadores o radicales- suelen dar del tema, es el de una sociedad perfectamente integrada donde uno desempeña el rol que le corresponde y goza de un status acorde, y en la que el conflicto (dominación, lucha de clases, etc.) está más o menos perfectamente regulado. En esta sociedad supuestamente equilibrada, los colectivos de inmigrantes aparecen de forma subrepticia e inmediatamente tienden a buscar su acomodo; a encontrar su nicho dentro de la nueva esfera social en que van a moverse de ahora en adelante. La obsesión por la integración de los inmigrantes parece haberse convertido en la pesadilla de los estudiosos del fenómeno migratorio, mucho más que de los propios inmigrantes (Castles y Kosack, 1973).

Y ello es así porque, a mi juicio, la mayor parte de los sociólogos actuales han adoptado un enfoque sistémico en su aproximación al concepto de la estructura social actual. Así, gran parte de los estudiosos que se dedican al tema del análisis de la inmigración, lo hacen desde una perspectiva «integracionista» del orden social; esta perspectiva implica el que queden integrados en el sistema general global fenómenos tales como la delincuencia, la desviación o la formación de ghettos, fenómenos todos ellos que la sociología tradicional de corte «durkheimiano" consideraba como outsiders o fuera del sistema, y la sociología marxista como una consecuencia estructural del sistema capitalista, pero no por ello menos marginal.

- Sobre la lógica situacional y su repercusión en la toma de decisiones sobre problemas concretos, véase S. GINER (1978). Para un análisis detallado del concepto de clase social en el estudio de las migraciones internacionales, véase J. SaLCEDO (1979).

- Un gran avance lo constituye el conocido artículo de LockwOod (1964), aunque en otro sentido al que he señalado más arriba. 
El pecado capital del «integracionismo sociológico» consiste en postular que la existencia de un orden social implica necesariamente la existencia de integración. Por supuesto que el orden consensual o pactado implica un mayor grado de cohesión y consenso que el orden coercitivo o impuesto, pero en ambos casos puede hablarse de una correlación positiva entre integración y orden social ${ }^{8}$, siempre desde ese punto de vista teórico.

La situación de los inmigrantes en nuestra sociedad industrializada actual tiende a clarificarse si utilizamos una perspectiva estructural diferente; en efecto, nada hay que nos obligue a considerar la sociedad global como un todo integrado donde hasta lo marginal o desviado sea funcional. Para determinados propósitos es incluso más útil considerar un modelo de sociedad basado en supuestos no integracionistas del orden y la estructura; así, la visión conflictivista se me antoja mucho más utilizable, ya que evita la adop. ción de algunos supuestos - como el de la integración-, que están en contradicción abierta con nuestra práctica cotidiana como entes que nos movemos en una realidad estructural conflictiva y desintegrada. La realidad parece ser más cercana a la descrita por Hobbes, donde el orden social nos viene impuesto por los grupos que detentan el poder político y económico de la sociedad, y al resto de los grupos o clases que nos movemos en ella no nos quedan sino dos alternativas: sujeción a, y observancia de la normativa impuesta (acompañada por un mecanismo más o menos eficaz para el cambio de la misma), o bien rebelión. Y lo normal (estadísticamente normal) son la observancia y la sujeción. Sujeción y observancia atenuadas por esas reglas no escritas de transgresión de la norma de que hablaba antes, y que actúan como válvula de seguridad del sistema en su conjunto.

Volviendo al tema que nos ocupa, veamos cuál es la situación de una colectividad inmigrante que se enfrenta (colectivamente) a la difícil tarea de la re-socialización. Y para no caer en la especulación, vamos a ceñirnos a un caso concreto: la inmigración pakistaní a Inglaterra, y de forma aún más concreta, los Sikbs en Leeds ${ }^{9}$. Los Sikbs, procedente del Punjab han estado llegando a Inglaterra desde la segunda mitad del siglo XIX, aunque el grueso de la inmigración ha tenido lugar en la década de los 1950, y algo menos en los 60. En su mayoría, eran jóvenes de clase media rural que originariamente emigraron con el único propósito de hacer dinero y volver luego a casa con un prestigio y un status superiores a los que tenían antes de partir.

* Sobre el tema del orden social en la Teoría Sociológica puede verse la magistral sistematización del profesor P. CoHEN' (1968). El orden social es para CoHEN el problema central de la Teoría Social, sea cual sea su signo y tendencia.

${ }^{9}$ Para un análisis detallado de los sikhs en Inglaterra pueden verse $\mathrm{R}$. y C. BALLARD (1977). Usualmente el sociólogo occidental tiende a simplificar en exceso su atención a las colectividades que no conoce; así suele hablarse de los inmigrantes españoles, portugueses o pakistanies, cuando lo cierto es que tales colectivos no existen. Las colectividades inmigrantes tienden a reproducir inicialmente el sistema de desigualdades existentes en su país de origen. Afortunadamente no es el caso del artículo de Roger y Catherine BaLciard. 
Cuando los sikbs comenzaron a llegar en masa a Inglaterra, adoptaron un sistema de organización social basado totalmente en el patronazgo; algunos compatriotas llegados con anterioridad se convirtieron en patrones (caseros, empleadores, prestamistas, etc.) de los recién llegados, que quedaron así en una situación de sometimiento total a unos conciudadanos suyos, que actuaban de intermediarios con la sociedad local. Inicialmente se adoptó el sistema de vivir en comunidades de hombres solos en una misma casa, por lo que los contactos con la sociedad local se redujeron al máximo; sin embargo, poco a poco se observó una daptación hacia las pautas de vida locales: abandono del turbante y el sari o túnica, occidentalización en el vestir, consumo de alcohol, etc. A partir de 1960, las mujeres sikbs (esposas e hijas de algunos de los ya residentes) comienzan a llegar a la isla; algunas familias se reúnen y se convierten en foco de atracción social (fiestas civiles y religiosas, etc.) de la nueva comunidad; desde este momento se imponen reglas más duras a la comunidad en su conjunto (abandono del alcohol, vuelta al uso del turbante, etc.), lo que supone, desde la perspectiva «integracionista» que he venido criticando, un retroceso en el proceso de integración. Este retroceso se manifiesta incluso en una supresión radical de las relaciones entre sikbs y mujeres locales, que son vistas como moralmente degeneradas por las costumbres tradicionales de los recién llegados; en palabras de uno de los interesados:

«Al principio, éramos novatos en todo. Trabajábamos mucho y vivíamos en condiciones difíciles, pero cuando nos divertíamos, realmente lo pasábamos bien. Teníamos mucha cerveza y también mujeres. Ahora que nuestras familias han llegado, todos somos muy estrictos. Muchos se han vuelto a poner el turbante y algunos incluso no beben ya» ${ }^{10}$.

En estas condiciones, la aplicación del concepto tradicional de integración, entendiendo por tal no la interacción de pautas, normas y costumbres entre la cultura inmigrante y la local, sino la adaptación de la primera a la segunda, con la pérdida de sus características propias en beneficio de la misma, no deja de ser una afirmación superficial de escasa relevancia sociológica. Porque lo cierto es que el concepto de integración, cuando se aplica a la situación que se da al entrar en contacto dos culturas bien diferenciadas, ha sido siempre sinónimo de asimilación o destrucción de una cultura por otra, y nunca la integración real; por ello, para la mayoría de los sociólogos europeos, sean de izquierdas o de derechas, la integración de los inmigrantes ha significado siempre la asimilación de la cultura inmigrante y la conversión de los recién llegados a la cultura social global o - -en el mejor de los casos- a la subcultura propia de la clase social (media u obrera generalmente)

10 Citado por R. y C. Ballard (1977). 
a la que pertenecían los inmigrantes. Y cuando esto no sucedía así, se hablaba de la dificultad de integrar a los nuevos habitantes, y se ponía de manifiesto cómo sus nietos (que ya no son inmigrantes) se integraban fácilmente, o cómo el problema tendía a resolverse con el tiempo (a medida que iba falleciendo la primera generación de inmigrados).

El propio André Gorz ha caído recientemente en esta trampa «integracionista» (Gorz, 1970). Y es que - y quiero destacar esto aun a riesgo de parecer repetitivo- intereses comunes no significan ni tienen por qué significar integración, y menos aún ese tipo de asimilación unidireccional de que he venido hablando, y que es la panacea que las diferentes sociedades ofrecen a los inmigrantes que a ellas arriban.

\section{I I}

Cuestión muy estrechamente relacionada con la de la intégración es la de la movilidad social, entendida como cambio de status. Como todo lo relacionado con el tema de la inmigración, se trata de un problema muy complejo que tiende a comparar niveles de status de dos sociedades muy diferentes: la de origen y la de adopción (temporal o final). En este sentido, Salvador Giner y yo señalábamos en un artículo reciente (1976) cómo

«el emigrante vive simultáneamente en dos universos sociales, con dos conjuntos de grupos de referencia; excepciones aparte, se considera a sí mismo mejor situado que sus iguales en su país de origen; pero también se ve peor situado que la gente local que 'debería' estar igual que él» (Giner y Salcedo, 1976, pág. 67).

Esta tendencia a la movilidad (mejora de status) es la que en gran medida explica gran parte de las migraciones de sociedades campesinas a áreas industrializadas (Watson, 1977), o las migraciones interiores en países, como España, con grandes desigualdades espaciales de renta, oportunidades y expectativas (Pérez Díaz, 1966).

La dualidad de universos en que se mueve el emigrante, y la presión a la que se ve sometido por la sociedad receptora condiciona sus respuestas a un medio hostil o indiferente (aunque los niveles materiales sean, en muchos casos, superiores a los que dejó atrás). Así, el campesino sin tierras que emigró para mejorar y que se ve rechazado por una sociedad que no comprende, se verá pronto obligado a elegir entre alguna de las alternativas siguientes:

1. Volverse a su tierra con los suyos. Este parece ser el caso más frecuente, aunque paradójicamente es aquel para el que existe menos información, ya que el sujeto ha dejado - técnicamente, al menos- de ser consi- 
derado un emigrante. En algunas sociedades está mal visto, ya que la emigración llega casi a ser contemplada como un rito de paso hacia la madurez, con un significado semejante a la pérdida de la virginidad en los varones (Philpott, 1977); sin embargo, la vuelta a casa, aunque al principio pueda ser humillante, es siempre reconfortante si el medio extraño es extremadamente hostil.

2. Permanecer, a pesar de todo, e «integrarse» en la sociedad local. Este ha sido el comportamiento tradicional de los emigrantes a otros países hasta fechas muy recientes. La carestía de los pasajes, junto con la presión de hijos y nietos obligaban al inmigrante a quedarse de fgorma definitiva en su país de adopción. La «integración» o asimilación en los grupos con cultura fuertemente interiorizada nunca fue total, como lo atestiguan las comunidades chinas o italianas en los Estados Unidos de América. Lo mismo puede decirse de la «inmigración forzosa» que supuso la esclavitud ".

3. Permanecer, alimentando el «mito del retorno». En nuestros días los costes de transporte se han abaratado, y las comunicaciones son mucho más fluidas, lo que proporciona posibilidades técnicas que no son demasiado desventajosas económicamente para la vuelta al lugar de origen. Por otra parte, es una práctica general el pago interestatal de seguridad social (pensiones de jubilación) a ciudadanos de un país que trabajan en otro y que vuelven al primero a pasar sus últimos años de vida. Esta práctica ya usual ha ayudado a potenciar la aparición del «mito del retorno», creencia muy generalizada entre los emigrantes según muchos estudiosos del tema. Esta «mitología del retorno» estaría basada en los siguientes aspectos:

a) El emigrante sigue pensando en relación con el universo de referencia que es su propia comunidad de origen. Como ya hemos señalado, su status ha mejorado con respecto al que tenía en ese lugar. Por ello no tiene nada de particular que vuelva al mismo haciendo ostentación de su nueva situación (la figura del «indiano», en nuestro país fue bien elocuente en el pasado). Esta nueva situación - estar mejor que sus iguales- hace que muchos emigrantes tiendan a idealizar su propia comunidad a través de una idealización de su nueva situación en esa comunidad. Ahora pertenecen a una nueva clase media local; invierten sus ahorros, compran casas, participan en obras comunitarias y hacen donativos cuantiosos para la iglesia, mezquita o templo local, con lo que su papel social ha experimentado una sobrevaloración; sobrevaloración que será mayor cuanto más pobre y atrasada sea su comunidad de origen.

b) El emigrante se sabe discriminado, rechazado e incluso marginado en el país de adopción. Aunque en su país de origen no tuviera ningún dere-

"No es mi itención comparar las migraciones laborales actuales con la esclavitud de los siglos xvi y xvir. Hay, sin embargo, algunos autores que tienden a establecer un paralelismo (BERger y MoHR, 1975) que está fuera de lugar. 
cho social o político, observa que está en una manifiesta inferioridad de condiciones con respecto a sus compañeros trabajadores locales. Colectivamente no puede organizarse, e individualmente deberá renunciar a su nacionalidad y cultura para gozar de una hipotética igualdad de derechos sociales y políticos. Y ello en los escasos lugares (Canadá, USA) donde la re-naturalización es posible. En general, en los países europeos el adquirir nacionalidad es muy difícil, y en algunos de ellos (Suiza) prácticamente imposible, salvo en el caso de grandes fortunas, profesionales muy cualificados o intelectuales preeminentes.

A todo lo anterior hay que crear el clima de inseguridad colectiva que ha creado la crisis económica generalizada durante nuestra década; en función de ella, el papel de los inmigrantes como tampón o amortiguador de las oscilaciones cíclicas de la crisis, es decir, como ejército industrial de reserva, no ha hecho sino agudizarse. El trabajador inmigrante se sabe, más que nunca, como un simple peón en el juego de intereses desatado entre patronales y sindicatos de los países ricos con la aquiescencia del Estado; sabe que por más declaraciones que hagan a su favor intelectuales comprometidos y centrales sindicales, los intereses de los trabajadores locales serán siempre antepuestos a los suyos, como la experiencia ha venido demostrando repetidamente a lo largo de los últimos veinte años.

En función de todo ello, y en particular por su compleja posición respecto de los dos grupos de referencia a que ha de volverse el emigrante para analizar su situación, los grupos de inmigrados reaccionan pensando en el retorno, que ha llegado a convertir en un mito con posibilidades técnicas de realización por vez primera. Pero, aunque el mito del retorno al «paraíso perdido» haya existido siempre, las posibilidades técnicas de su realización, derivadas de la tecnología y de la moderna capacidad de gestión, tienen una repercusión social de gran alcance: el rechazo de la integración por parte de los inmigrantes.

En efecto, «si la sociedad 'adoptiva' tiende a rechazarme; si se trata de un sistema donde siempre voy a llevar la peor parte; si he incrementado mi posición respecto de los amigos que quedaron allá; si puedo volver un día y además voy a volver... ¿Para qué me voy a integrar?». Este puede ser el resumen de las manifestacione $s$ formuladas por distintos grupos de inmigrantes, sobre todo asiáticos y caribeños en Inglaterra, en los últimos años. El resultado inmediato ba sido un incremento de la conciencia colectiva como grupos diferenciados étnica,. cultural y socialmente.

Algunos sociólogos radicales (Catles y Kosack; 1973) persuadidos de la bondad de la existencia de una clase obrera universal, unida por lazos de solidaridad y conciencia de clase bien definidos han criticado esta tendencia hacia la automarginación como de «políticamente reaccionaria y socialmente peligrosa». En efecto, la existencia de «secciones» o clusters dentro de un 
cuerpo social antaño separado únicamente por divisiones de clase o nacionales es un fenómeno nuevo en la sociedad europea, cuyos alcances futuros son aún imprevisibles. Sin embargo, el fenómeno - reaccionario o no- se está dando con diferente intensidad en gran parte de los países europeos occidentales industrializados. El fenómeno de la inmigración laboral está introduciendo, pues, modificaciones en la estructura social, sobre las que por el momento sólo cabe la especulación.

\section{V}

En un artículo reciente, que ya he citado anteriormente, Salvador Giner y yo construimos una tipología clasificatoria de los tipos de respuesta del emigrante hacia la sociedad receptora ${ }^{12}$. De los cuatro tipos de respuesta básicos que allí se analizaban, el de subordinación pasiva, consistente en una «ausencia de integración en el sistema de clases de la sociedad, combinada con una aceptación generalizada de las pautas de dominación y autoridad estblecida» (p. 65) era la respuesta «normal» que los inmigrantes daban al sistema establecido. Su subordinación y su no integración de clase venía analizada en función de que los inmigrantes eran «importados por el sistema económico sobre una base que les perpetúa como seres extraños al mismo: son trabajo alquilado, personal prestado y lo siguen siendo" (p. 65).

Esta subordinación pasiva, sobre una base de «prestación temporal de servicios» (!) es la menos conflictiva posible. Pero no hay que ser un futurólogo avezado para darse cuenta de que la situación va a cambiar en un corto espacio de tiempo. Y la dirección del cambio va a depender de la aceptación o el recbazo activo de las pautas de dominación y autoridad establecidas. Partiendo de la hipótesis de rechazo activo que es la más plausible, dado el grado de desconexión de los inmigrantes respecto de la estructura social de los países receptores, y de su conciencia clara de estar en situación

12 La tipología surgía de combinar los niveles integración de clase y subordinación política, de la forma siguiente:

SUBORDINACION POLITICA

\begin{tabular}{|c|c|c|c|}
\hline & & $(+)$ & $(-)$ \\
\hline INTEGRACION & $(+)$ & $\begin{array}{l}\text { ASIMILACION } \\
\text { POSITIVA }\end{array}$ & $\begin{array}{l}\text { INSUBORDINACION } \\
\text { SOLIDARIA }\end{array}$ \\
\hline CLASE & $(-)$ & $\begin{array}{l}\text { SUBORDINACION } \\
\text { PASIVA }\end{array}$ & $\begin{array}{l}\text { INSUBORDINACION } \\
\text { PARTICULARISTA }\end{array}$ \\
\hline
\end{tabular}

para mayor detalle véase el artículo citado (GINER y SALCEDo, 1976) o bien su versión reformada más reciente (ibídem, 1978). 
de inferioridad real respecto de sus «iguales» teóricos, parece bien evidente que se van a dar los dos tipos de respuesta siguientes:

a) Insubordinación solidaria. Implica un alto grado de integración de clase. Se dará entre aquellos colectivos inmigrantes que no tiendan a la formación de clusters o comunidades separadas y no integradas, del tipo de las. que he señalado con anterioridad. Se trata del tipo de conflicto que plantearán los trabajadores procedentes de países de la CEE que trabajen en otros países comunitarios (italianos en Alemania, por ejemplo).

b) Insubordinación particularista. Fue caracterizado este tipo de respuesta como aquella que «tiene lugar cuando una colectividad inmigrante inicia de forma aislada algún tipo de rechazo abierto del sistema de dominación inicialmente cuando tuvo lugar la emigración; el rechazo no está coordinado ni suele ser compartido por los otros trabajadores» (p. 66). De hecho sería el tipo de acción política característica de comunidades inmigrantes marginadas, y que es proclive a desembocar en formas de terrorismo y acción política violenta. Los sud-moluqueños en Holanda es un ejemplo, aunque en este caso hay un planteamiento político de promesas incumplidas y un pasado colinal; sin embargo, no es difícil encontrar características peculiares determinantes en cualquiera de los grupos inmigrantes marginados (pakistanies en Inglaterra, argelinos en Francia, turcos en Alemania).

Una tercera posibilidad es la tendencia al autogobierno y la adopción de formas políticas propias por parte de las comunidades inmigrantes, caso de ser suficientemente numerosas y ocupar un espacio físico determinado. Esta tercera posibilidad es la más remota y utópica, pero no por ello hay que perderla de vista, ya que puede llegar a plantearse en un futuro no muy lejano ${ }^{13}$.

De todo lo señalado anteriormente se desprende una conclusión general: la ineficacia sociológica de las concepciones tradicionales acerca de los procesos migratorios en general y sobre el asentamiento de los emigrantes en particular. Esta ineficacia parece ser debida al desconocimiento de los cambios que han tenido lugar en los últimos años en la estructura social de los países industrializados; desconocimiento que se traduce generalmente en la creencia en procesos o estructuras que bien ya no existen o que se han modificado hasta hacerse irreconocibles. Como muestra se podría tomar la creciente corporatización de la sociedad industrial, que afecta hasta a sus sectores más marginados, $\mathrm{y}$ que aún no ha sido objeto de estudio detenido.

También parte de esa ineficacia que he señalado se debe al método de estudio empleado. El individualismo metodológico, tan denostado ya en nuestros días, ha presidido gran parte de los análisis que se han realizado en torno

'3e hecho se ha planteado ya en algunos casos (colonias alemanas en Brasil, Uruguay y Chile), aunque con un alcance limitado y sin resultado alguno hasta el momento. 
a los problemas de la emigración, con independencia de la perspectiva teórica de partida. Se ha olvidado con demasiada frecuencia que los procesos de adaptación son colectivos, como ya señalaron Park y Burgess en los años veinte y Engels en 1880 . Se ha olvidado también la importancia de procesos de segregación y autosegregación étnica y cultural en función de un complejo formado por la clase social, la cultura y la raza. Sin embargo, se dispone ya de un acopio de material empírico considerable, por lo que es de suponer que la próxima década va a ser testigo de fuertes avances teóricos en el estudio de las migraciones.

Post SCriptum.-Algunas de las ideas avanzadas en las páginas anteriores han sido expuestas públicamente ante los profesores del Departamento de Sociología de la Universidad de Barcelona (Pedralbes). De la discusión de las mismas se llegó al punto álgido de su aplicabilidad al caso español y, de forma expresa, a la inmigración a Cataluña de trabajadores procedentes del resto de España.

Hay que señalar cómo la problemática básica es diferente: no se trata de una emigración internacional y, por tanto, los problemas de participación política y sindical planteados por esas migraciones desaparecen. Sin embargo, y a mi juicio buena parte de la problemática derivada de los conflictos culturales y del binomio integración-asimilación, es aplicable al caso catalán. Sin embargo, y aunque desde el punto de vista teórico se puedan avanzar algunas ideas, se carece aún de la información necesaria para comprobarlas de forma empírica. Así, la investigación realizada por C. Solé y otros (C. Solé, 1980) puede ser de gran utilidad siempre y cuando hagan públicas las transcripciones de las entrevistas en profundidad realizadas. Ello evitaría interpretaciones simplistas de los fenómenos de resistencia a la asimilación, tanto en el pasado (despectivamente descalificados como «lerrouxismo») o en el presente (presencia del PSA en el Parlamento catalán). 


\section{BIBLIOGRAFIA CITADA}

Ballard, R. y C. (1977): "The Sikhs: The development of south Asian Settlements in Britain", en Watson, J. L.: Between Two cultures. Oxford: Basil Blackwell, págs. 21-56.

Berger, J., y MOHR. J. (1975): A seventh man. Harmondsworth: Penguin.

Bernard, Ph. (1975): Les travailleurs étrangers en Europe Occidentale. París: Mouton.

Castres, S., y Kosack, G. (1973): Inmigrant workers and social structure in Western Europe. Oxford: The University Press.

CETEDIM (1975): Les immigrés. París: Stock II.

Cohen, P. (1968): Modern Social Theory. Londres: Methuen.

GINER, S., y SALCEdo, J. (1976): "Un vacío teórico: la explicación causal de la migración", en Agricultura y Sociedad, n. ${ }^{\circ}$ 1, págs. 113-126.

GINER, S., y SALCEDO, J. (1978): "Workers immigration and social structures in Europe", en S. GINer y ARcher, M. S.: Contemporary Europe. Londres: Routledge and Kegan Paul.

GoRz, A. (1970): "Immigrant Labour", en New Left Review, n..$^{\circ}$ 61, págs. 28-31.

LASA, J., y Martinez, P. (1970): Síndromes psicopatológicos condicionados por la inmigración y la emigración. San Sebastián: Diputación provincial.

PAscun, A. (1970): El retorn dels emigrants. Barcelona: Nova Terra.

Phrlpotr, S. (1977): “The Montserratians", en Watson, J, L.: Between..., cit., páginas $90-119$.

Rose, A. (1972): "The integration of People", en M. Hodges (ed.): European Integration. Hardmonsworth: Penguin.

SoLE, C. (180): "La identificación de los emigrantes con la cultura catalana", en REIS, n. 9, págs. 119-138. 